\title{
THE SOLUTIONS OF THE QUASILINEAR KELLER-SEGEL SYSTEM WITH THE VOLUME FILLING EFFECT DO NOT BLOW UP WHENEVER THE LYAPUNOV FUNCTIONAL IS BOUNDED FROM BELOW
}

\author{
TOMASZ CIEŚLAK \\ Institute of Mathematics, Polish Academy of Sciences \\ Śniadeckich 8, P.O. Box 21, 00-956 Warszawa, Poland \\ E-mail: T.Cieslak@impan.gov.pl
}

\begin{abstract}
In [2] we proved two kinds of mechanisms of preventing the blow up in a quasilinear non-uniformly parabolic Keller-Segel systems. One of them was a priori boundedness from below of the Lyapunov functional. In fact, we were able to present a condition under which the Lyapunov functional is bounded from below and a solution exists globally. In the present paper we prove that whenever the Lyapunov functional is bounded from below the solution exists globally.
\end{abstract}

1. Introduction and preliminaries. In what follows we investigate the non-uniformly parabolic boundary value problem

$$
\begin{aligned}
\frac{\partial u}{\partial t} & =\nabla \cdot[\alpha(u) \nabla u-u \beta(u) \nabla v] \text { in } U \times(0, T), \\
\frac{\partial v}{\partial t} & =D_{v} \Delta v-v+u \text { in } U \times(0, T), \\
\nabla u \cdot \vec{n} & =0, \quad \nabla v \cdot \vec{n}=0 \text { on } \partial U \times(0, T), \\
u(x, 0) & =u_{0}(x), \quad v(x, 0)=v_{0}(x) \text { in } U,
\end{aligned}
$$

where $U$ is a domain of $\mathbb{R}^{n}$ with a sufficiently smooth boundary. By $\vec{n}$ we denote the outer normal vector.

The problem is studied under the following hypotheses:

T: (i) There exists $\varepsilon>0$ such that $\beta \in C^{2}(-\varepsilon, \infty)$ is a positive bounded function and $D_{v}$ appearing in (2) is a positive constant.

2000 Mathematics Subject Classification: 92C17, 35K60, 35K57.

Key words and phrases: chemotaxis equations, global-in-time existence and uniqueness.

The paper is in final form and no version of it will be published elsewhere. 
(ii) There exists $\varepsilon>0$ such that $\alpha \in C^{2}(-\varepsilon, \infty)$ is a positive bounded function.

(iii) The function $\alpha$ converges to 0 when its arguments tend to $\infty$.

Because of $\mathbf{T}$ (iii) we say that (1)-(4) is non-uniformly parabolic.

In [2] we showed two mechanisms of preventing the blow-up. In particular, we proved the following result [2, Theorem 2]. Assume $n=2,3$, and there exist $M>0$ and $N>0$ such that

$$
\begin{gathered}
\frac{\beta(u)}{\alpha(u)} \leq M, \\
\frac{u \beta(u)}{\alpha(u)} \leq N u^{\gamma},
\end{gathered}
$$

where $\gamma<\frac{1}{2}$ if $n=2$ and $\gamma<0$ if $n=3$. Then there exists a unique global-in-time classical solution to (1)-(4) provided $u_{0}, v_{0} \in W^{1, p}(U), p>n$.

One can notice that for $u>1$ the assumption (6) covers (5). Moreover, the proof of $[2$, Theorem 2] can be easily modified in order to prove the theorem assuming only (6) holds for $u>1$. We were not paying our attention to this because of the biological motivation to our considerations. We wanted to find the conditions on $\alpha$ and $\beta$ under which the densities of cells in the so-called Hillen-Painter models do not explode in finite time. In [5] the authors introduced, basing on biased random walks, the models of chemosensitive movement that take into account the volume filling effect. The tool to do it was the assumption that the probability $q$ that a cell attains the position $(x, t)$ depends on the density of cells at this position, which reflects the finite size of cells. Such an approach results in (1)-(4) with $\alpha$ and $\beta$ given in the following way:

$$
\alpha(u)=q(u)-u q^{\prime}(u) \text { and } \beta(u)=q(u) .
$$

One may obtain two kinds of situation, there is a threshold value $u_{0}$ at which $q=0$ or not. In the second situation one might arrive at $\mathbf{T}$ (iii) and non-uniformly parabolic system must be considered. But notice that even in this case (5) holds with $M=1$ if we assume that $q$ is a nonincreasing function of the density of cells. That is why we did not try to justify our theorems considering conditions on $\alpha$ and $\beta$ only for $u>1$. In the following paper we still do not do it, but once again we leave it to the reader to see that our considerations are true assuming only (6) for $u>1$ instead of (5), (6).

Notation. The norm in the space $L^{p}(U), 1 \leq p \leq \infty$, is denoted by $\|\cdot\|_{p}$. The same notation is used for vector valued functions $u \in L^{p}\left(U: \mathbb{R}^{n}\right)$. Classical Sobolev spaces will be denoted by $W^{1, p}(U)$ for $1 \leq p \leq \infty$.

In this paper we prove a stronger version of [2, Theorem 2]. We assume only $\gamma<\frac{2}{n}$. Before starting the discussion about the connections of our result and the boundedness from below of the Lyapunov functional for (1)-(4), let us mention that for $\alpha=\beta=1$ we arrive at the so-called minimal version of Keller-Segel system [8]. It was the first model proposed as a mathematical description of chemosensitive movement. But in this system blow-ups could occur (see [7], [4]). In order to avoid this property, the Hillen-Painter models were introduced in [5]. 
Let us turn our attention to the Lyapunov functional for the system (1)-(4). We define the function

$$
\Phi(s):=\int_{0}^{s} \int_{1}^{\sigma} \frac{\alpha(\tau)}{\tau \beta(\tau)} d \tau d \sigma, \quad s>0 .
$$

Then it turns out by [2, Lemma 2.1] that

satisfies

$$
L(u, v):=\int_{U} \Phi(u)+\frac{D_{v}}{2} \int_{U}|\nabla v|^{2}+\frac{1}{2} \int_{U} v^{2}-\int_{U} u v
$$

$$
\frac{d}{d t} L \leq 0
$$

By [6, Lemma 5.2] we have

LEMMA 1.1. If (6) holds with $\gamma<\frac{2}{n}, n \geq 2$ then there exists a constant $C$ such that

$$
L \geq C
$$

for every $(u, v) \in\left(W^{1, \infty}(U)\right)^{2}$. Moreover, if there exists $M$ such that

$$
\frac{u \beta(u)}{\alpha(u)} \geq M u^{\gamma}
$$

for $\gamma>\frac{2}{n}(n=2,3)$ then for any fixed $\lambda>0$ there exists $\varepsilon_{0}>0$ and families $\left(u_{\varepsilon}\right)_{\varepsilon \in\left(0, \varepsilon_{0}\right)} \subset W^{1, \infty}$ and $\left(v_{\varepsilon}\right)_{\varepsilon \in\left(0, \varepsilon_{0}\right)} \subset W^{1, \infty}$ such that $u_{\varepsilon}>0$ and $v_{\varepsilon}>0$ in $\bar{U}$,

but

$$
\int_{U} u_{\varepsilon}=\lambda \text { and } \int_{U} v_{\varepsilon} \rightarrow 0 \text { as } \varepsilon \rightarrow 0
$$

$$
L\left(u_{\varepsilon}, v_{\varepsilon}\right) \rightarrow-\infty, \varepsilon \rightarrow 0 .
$$

Let us also point out [6, Remark after Lemma 5.1] that from the boundedness from below of the Lyapunov functional (see Lemma 1.1) we are able to infer a priori bounds on $u$. Indeed, we have

Lemma 1.2. Assume (6) holds with $\gamma<\frac{2}{n}$. Then there exists $N>0$ such that

$$
\sup _{t \in[0, T]}\|u(\cdot, t)\|_{2-\gamma}<N .
$$

We shall need the following theorem that enables us to handle the non-uniformly parabolic equation [3, Theorem 2.2]. This theorem was also proved in [1] with the use of completely different methods.

Theorem 0. Let $0<T<\infty, \nabla v \in L^{\infty}(U \times(0, T))$, (5) and T. Assume that $u$ is a $L^{2}$-weak solution to

$$
\begin{aligned}
\frac{\partial u}{\partial t} & =\nabla \cdot[\alpha(u) \nabla u-u \beta(u) \nabla v] \text { in } U \times(0, T), \\
\nabla u \cdot \vec{n} & =0, \quad \nabla v \cdot \vec{n}=0 \text { on } \partial U \times(0, T), \\
u(x, 0) & =u_{0}(x) \text { in } U,
\end{aligned}
$$

corresponding to $u_{0} \in L^{\infty}(U)$. Then

$$
\sup _{\left[0, T_{\max }\right) \cap[0, T]}\|u(\cdot, t)\|_{\infty}<\infty,
$$

where $T_{\max }$ is the maximal existence interval of the solution. 
We emphasize that Theorem 0 says only that

$$
u \in L_{l o c}^{\infty}\left((0, \infty), L^{\infty}(U)\right) .
$$

We cannot infer the uniform in time boundedness of a solution from this property.

The following lemma will be also of importance in the further part of the paper $[2$, Lemma 2.3].

Lemma 1.3. Let $v$ be the solution to (2), $T<\infty$.

(i) For $q=\frac{n \delta}{n-\delta}, \delta \geq 1$ and some positive constant $C$ we have

$$
\sup _{t \in(0, T]}\|\nabla v(\cdot, t)\|_{q} \leq C \sup _{t \in(0, T]}\|u(\cdot, t)\|_{\delta} .
$$

(ii) Moreover, if $\sup _{t \in(0, T]}\|u(\cdot, t)\|_{p}<\infty, p>n$, then

$$
\sup _{t \in(0, T]}\|\nabla v(\cdot, t)\|_{\infty}<\infty .
$$

2. Main result. By $C$ we denote generic constants whose value may vary from line to line. Let us prove the following

TheOREM 2.1. We consider the case $n=2$, 3. Assume (5), (6) for $\gamma<\frac{2}{n}$ and $\mathbf{T}$. Then there exists a unique classical nonnegative global-in-time solution to (1)-(4) corresponding to nonnegative initial data $u_{0}, v_{0} \in W^{1, p}(U), p>n$.

Let us first prove a lemma which will be crucial in the proof of Theorem 2.1. This lemma is a generalization of the one presented in [6, Lemma 4.4] to the case of quasilinear non-uniformly parabolic system. In fact, it uses only (1) to derive from given estimates of $u$ and $v$, a better one on $u$.

LEMMA 2.2. Suppose for given $v$ such that

$$
\sup _{[0, T]}\|\nabla v(\cdot, t)\|_{q_{0}} \leq C,
$$

$u$ is the solution to (1) under no-flux boundary condition. Assume also

$$
\sup _{[0, T]}\|u(\cdot, t)\|_{\gamma_{0}} \leq C .
$$

Moreover suppose

$$
\left(\frac{n}{q_{0}}-1\right) \gamma_{0}<n(1-\gamma) .
$$

Then for any $\gamma_{1}>\max \left\{\gamma_{0}, 2-2 \gamma\right\}$ which fulfills

$$
\begin{gathered}
\left(\frac{n}{q_{0}}-1\right) \gamma_{1}<(n-2)(1-\gamma) \\
\sup _{[0, T]}\|u(\cdot, t)\|_{\gamma_{1}} \leq C .
\end{gathered}
$$

Proof of Lemma 2.2. From the proof of [6, Lemma 4.4] we infer that in order to prove Lemma 2.2 it is enough to show the analogous inequality to $[6,(35)]$. To this end we use 
the ideas that appeared in [3], [1], and introduce the nonlinear convex functional $\phi_{p}(u)$ satisfying both $\phi_{p}(0)=0$ and $\phi_{p}^{\prime}(0)=0$ by

$$
p(p-1) \eta^{p-2}=\phi_{p}^{\prime \prime}(\eta) \alpha(\eta) .
$$

for every $\eta>0$ and $p>1$. Notice that

$$
\int_{\Omega} \eta^{p} d x \leq C \int_{\Omega} \phi_{p}(\eta) d x
$$

The inequality can be easily derived integrating twice (17).

We test (1) by $\phi_{\gamma_{1}}^{\prime}(u)$ and with the use of (17), (6) and the Young inequality we obtain

$$
\frac{1}{\gamma_{1}} \frac{d}{d t} \int_{U} \phi_{\gamma_{1}}(u) d x+C \int_{U}\left|\nabla u^{\frac{\gamma_{1}}{2}}\right|^{2} d x \leq C \int_{U} u^{2 \gamma+\gamma_{1}-2}|\nabla v|^{2} d x .
$$

Thus, writing $w$ in the place of $u^{\frac{\gamma_{1}}{2}}$, integrating (19) in time and then applying (18) to the left-hand side and Hölder's inequality to the right-hand side we arrive at

$$
\begin{aligned}
& \frac{1}{\gamma_{1}} \int_{U} w(T)^{2} d x-\frac{1}{\gamma_{1}} \int_{U} w(0)^{2} d x+\int_{0}^{T} \int_{U}|\nabla w|^{2} d x d t \\
& \leq C \int_{0}^{T}\left(\int_{U}|\nabla v|^{q_{0}} d x\right)^{\frac{2}{q_{0}}}\left(\int_{U} w^{\frac{2 q_{0}\left(2 \gamma+\gamma_{1}-2\right)}{\left(q_{0}-2\right) \gamma_{1}}} d x\right)^{\frac{q_{0}-2}{q_{0}}} d t .
\end{aligned}
$$

In view of (13) the right-hand side of the last inequality can be estimated by

$$
C \int_{0}^{T} \| w(\cdot, t) \frac{\frac{2\left(2 \gamma+\gamma_{1}-2\right)}{\gamma_{1}}}{\frac{2 q_{0}\left(2 \gamma+\gamma_{1}-2\right)}{\left(q_{0}-2\right) \gamma_{1}}} d t .
$$

Thus, the desired inequality, analogous to $[6,(35)]$, is proved.

Proof of Theorem 2.1. By [2, Theorem 2.1] we have the classical unique solution that is global provided $\|u\|_{\infty}$ is finite on finite time intervals. We see that by Theorem 0 we only need to estimate $\sup _{(0, T]}\|\nabla v(\cdot, t)\|_{\infty}$ for every finite $T$. But by Lemma 1.3 (ii) this is implied by (14) with $\gamma_{0}>n$ for every finite $T$.

By Lemma 1.2 we see that (9) holds. Then for dimension $n=2$ thanks to Lemma 1.3 (i) we estimate $(13)$ holds for $q_{0}>2$. Thus by Lemma 2.2 , since

$$
\left(\frac{n}{q_{0}}-1\right)<0
$$

which implies (16) for arbitrary finite $\gamma_{1}$, one concludes that (14) holds for $\gamma_{0}>2$.

For dimension $n=3$ much more sophisticated bootstrapping must be used. Fortunately, we can use the one introduced in [6, the proof of Lemma 4.5]. For the details we refer the reader to [6], here we only give the sketch of the method.

We introduce the sequence defined recursively by

$$
a_{k}=\frac{(n-2)(1-\gamma)}{n-2 a_{k-1}} a_{k-1}
$$

if $a_{k-1}<\frac{n}{2}, \infty$ otherwise. This is an increasing sequence provided we begin with $a_{0}>$ $2-\gamma$, and then there exists a finite $k_{0}$ such that $a_{k_{0}}=\infty$. Then we introduce the 
sequences $\varepsilon_{k}>0$ and $b_{k}$ such that

$$
b_{k}=\frac{(n-2)(1-\gamma)}{n-2 b_{k-1}} b_{k-1}-\varepsilon_{k}
$$

has the same properties as $a_{k}$, in particular there is $k_{0}$ such that $b_{k_{0}}>\frac{n}{2}$. We also define

$$
\bar{q}_{k-1}:=\frac{n b_{k-1}}{n-b_{k-1}} .
$$

By $\left[6,(42)\right.$ and (43)] we see that there exist $q_{0}, \ldots, q_{k_{0}}$ such that

$$
2<q_{k-1}<\bar{q}_{k-1}, \quad\left(\frac{n}{q_{k-1}}-1\right) b_{k-1}<n(1-\gamma)
$$

and

$$
\left(\frac{n}{q_{k-1}}-1\right) b_{k}<(n-2)(1-\gamma)
$$

Lemma 1.2 gives us the startpoint of the bootstrap procedure that allows us to conclude that (14) holds for $\gamma_{0}>\frac{n}{2}$. Indeed, beginning with (9) we have $b_{0}>2-\gamma$ and estimating successively $\sup _{(0, T]}\|\nabla v\|_{q_{k}-1}$ thanks to Lemma 1.3 (i), and then $\sup _{(0, T]}\|u\|_{b_{k}}$ thanks to Lemma 2.2, (21) and (22), finally we arrive at (14), $\gamma_{0}>\frac{n}{2}$ since there exists $b_{k_{0}}>\gamma_{0}>\frac{n}{2}$. But then once again we use Lemma 1.3 (i) in order to estimate $\sup _{(0, T]}\|\nabla v\|_{q}, q>n$. Then by Lemma 2.2 (with $q$ instead of $q_{0}$ in (16)), since (20) holds for arbitrary finite $\gamma_{1}$, we obtain (14) with $\gamma_{0}>n$, and the proof is finished.

\section{References}

[1] V. Calvez and J. A. Carrillo, Volume filling effects in the Keller-Segel model: energy estimates preventing blow-up, preprint.

[2] T. Cieślak, Global existence of solutions to a chemotaxis system with volume filling effect, preprint.

[3] T. Cieślak, Quasilinear non-uniformly parabolic system modelling chemotaxis, accepted for publication in J. Math. Anal. Appl.

[4] M. A. Herrero and J. J. L. Velázquez, A blow-up mechanism for a chemotaxis model, Ann. Scuola Norm. Sup. 24 (1997), 633-683.

[5] T. Hillen and K. Painter, Volume filling and quorum sensing in models for chemosensitive movement, Canadian J. Appl. Math. Quarterly 10 (2002), 501-543.

[6] D. Horstmann and M. Winkler, Boundedness vs. blow-up in a chemotaxis system, J. Diff. Eq. 215 (2005), 52-107.

[7] W. Jäger and S. Luckhaus, On explosions of solutions to a system of partial differential equations modelling chemotaxis, Trans. Amer. Math. Soc. 329 (1992), 819-824.

[8] E. F. Keller and L. A. Segel, Initiation of slime mold aggregation viewed as an instability, J. Theor. Biology 26 (1970), 399-415. 